Meta

Journal des traducteurs

Translators' Journal

\title{
Les clauses d'exonération de responsabilité : stratégies de traduction
}

\section{Carmen Bestué Salinas}

Volume 55, numéro 2, juin 2010

URI : https://id.erudit.org/iderudit/044241ar

DOI : https://doi.org/10.7202/044241ar

Aller au sommaire du numéro

\section{Éditeur(s)}

Les Presses de l'Université de Montréal

\section{ISSN}

0026-0452 (imprimé)

1492-1421 (numérique)

Découvrir la revue

\section{Citer cet article}

Bestué Salinas, C. (2010). Les clauses d'exonération de responsabilité :

stratégies de traduction. Meta, 55(2), 287-308. https://doi.org/10.7202/044241ar

META

Résumé de l'article

Le présent article traite d'une clause spécifique au droit anglo-saxon, celle de l'exonération de responsabilité. Probablement en raison de l'influence croissante de l'usage de l'informatique et, en particulier, d'Internet, le format et la structure des clauses rédigées en français ou espagnol imitent de plus en plus souvent le modèle anglo-saxon. Ce phénomène est, sans aucun doute, le fruit de la traduction. L'article vise à montrer, dans une perspective fonctionnaliste, que la méthode de traduction suivie et les techniques appliquées doivent tenir compte non seulement de critères juridiques ou linguistiques, mais aussi d'autres paramètres pragmatiques comme les facteurs de communication et les facteurs culturels. 


\title{
TERMINOLOGIE ET LINGUISTIQUE
}

\section{Les clauses d'exonération de responsabilité: stratégies de traduction}

\author{
CARMEN BESTUÉ SALINAS \\ Universitat Autònoma de Barcelona, Barcelone, Espagne \\ carmen.bestue@uab.cat
}

\begin{abstract}
RÉSUMÉ
Le présent article traite d'une clause spécifique au droit anglo-saxon, celle de l'exonération de responsabilité. Probablement en raison de l'influence croissante de l'usage de l'informatique et, en particulier, d'Internet, le format et la structure des clauses rédigées en français ou espagnol imitent de plus en plus souvent le modèle anglo-saxon. Ce phénomène est, sans aucun doute, le fruit de la traduction. L'article vise à montrer, dans une perspective fonctionnaliste, que la méthode de traduction suivie et les techniques appliquées doivent tenir compte non seulement de critères juridiques ou linguistiques, mais aussi d'autres paramètres pragmatiques comme les facteurs de communication et les facteurs culturels.
\end{abstract}

\begin{abstract}
This article considers a category of legal clause typical of English and American law, namely the exoneration clause. Both Spanish and French contracts increasingly replicate the layout and structure of equivalent clauses in English-language documents. This, doubtless, is a byproduct of English to Spanish translation and may have been a result of the growing impact of computer tools and especially the Internet. Our aim is to demonstrate, according to a functionalist approach, that the translation strategy and techniques used should factor in not only legal and linguistic criteria but also pragmatic parameters such as communicative and cultural elements.
\end{abstract}

\section{MOTS-CLÉS/KEYWORDS}

traduction juridique, droit comparé, contrats, dommages-intérêts, exonération de responsabilité

legal translation, comparative law, contracts, damages, exemption of responsibility

\section{Introduction}

L'utilisation courante de l'informatique par les consommateurs et les professionnels a fait proliférer un nouveau type de contrat, dont la signature se trouve accélérée parce qu'elle se fait facilement par un simple clic de souris. Ce qui, hier, caractérisait la partie des contrats écrite en minuscules ou reléguée aux annexes - ce que le signataire ne lisait jamais - s'étend, désormais, à la totalité des stipulations. Celles-ci défilent à une vitesse telle qu'il est pratiquement impossible de les lire. Malgré cela, on finit toujours par valider la case: «oui, j'accepte». De plus, le fait que ces contrats sont dans une grande majorité des cas des traductions de l'anglais représente 
une complexité supplémentaire, ce qui reste, en toute logique, ignoré par le consommateur.

Dans ce contexte, l'article a pour objectif d'analyser l'impact de la fonction du texte traduit sur les choix de traduction au sein de ces contrats et de souligner les défis que les termes culturellement marqués représentent pour le traducteur. Suivant la distinction établie par Nord (1997), et qui a été appliquée d'une façon remarquable par Dullion (2007) dans le cadre de la traduction des textes législatifs, nous allons étudier la traduction des clauses d'exonération en tentant de structurer les choix de traduction selon la fonction principale du contrat dont ces clauses relèvent. En effet, dans le cadre du fonctionnalisme, Nord (1997: 45-52) fait la distinction entre la traduction-document, où l'objectif est de renseigner le destinataire sur le texte de départ en tant que support d'un acte de communication qui a eu lieu dans la situation de départ, et la traduction-instrument qui a pour but de fournir le support d'un nouvel acte de communication. Dans le cadre que nous présentons ici, l'élément-clé qui nous sert à déterminer la fonction principale de la traduction des contrats est le droit du pays qui doit régir ledit contrat.

Afin d'exposer d'une façon plus concrète l'impact de la fonction du texte traduit dans les choix de traduction, nous avons sélectionné la clause suivante, qui provient d'un contrat de licence d'utilisation de programmes d'ordinateur de source privée. Néanmoins, des contenus similaires peuvent se trouver facilement sur Internet (la mise en relief en gras est nôtre):

In no event shall $\mathrm{xxx}$ or its licensors be liable for any special, indirect, incidental, consequential, or punitive losses or damages (including losses or damages for any lost revenues, profits, or data), even if advised or made aware of the possibility of any such losses or damages and regardless of whether the claim: (a) relates to any xxx software product, any third party software product, or the performance or non-performance thereof, or (b) is based on breach of contract or warranty, negligence or other tort, strict liability, or other theory of liability. The foregoing shall not limit xxx's liability as otherwise expressly set forth in article 8.0 nor xxx's liability for any breach of its obligations under section 5.2.3[...] (Document privé, adapté par l'auteure).

Les clauses d'exonération ou de limitation de responsabilité reçoivent des appellations différentes dans les contrats en anglais : disclaimer, limitation, exoneration or exlusion of damages, no warranties, waiver, etc., mais en définitive, le but recherché est d'exclure, ou du moins, de limiter la responsabilité de l'une des parties (en général, celle qui est en position de force) quant à la réparation des différents dommages qui pourraient survenir. Concrètement, on trouve habituellement des mentions telles que direct, indirect, consequential, incidental and special damages, qui énumèrent les dommages concernés. S'il est vrai que ces clauses se retrouvent naturellement dans les contrats rédigés en anglais, régis par le droit d'un pays de la common law auquel elles sont spécifiques, il n'est pas moins vrai qu'elles apparaissent de plus en plus souvent dans les contrats rédigés dans d'autres langues comme le français, l'italien ou l'espagnol, et qui sont soumis aux droits nationaux respectifs. Ces clauses sont fréquentes en particulier dans les contrats informatisés souscrits par Internet. Ces contrats ont même reçu la dénomination de e-contracts. Du fait qu'ils appartiennent au droit informatique, domaine juridique de création récente et d'accès facile à tous, ils sont particulièrement perméables à l'influence de la culture juridique anglosaxonne. Dans le domaine du droit informatique et, plus généralement, du droit de 
la propriété intellectuelle, nous retrouvons non seulement de nouvelles formes contractuelles, mais aussi des figures et des concepts juridiques créés aux États-Unis qui sont inconnus dans le pays où le texte traduit doit prendre effet.

Nous développerons, sous l'angle traductionnel, l'analyse de ces clauses en deux étapes. Dans un premier temps, nous verrons dans quel contexte elles s'insèrent, c'est-à-dire les contrats, et quelle est leur fonction au sein de ces derniers. Dans un deuxième temps, nous nous pencherons sur les problèmes de terminologie que ces clauses renferment.

\section{Analyse du contexte}

Le concept de contrat nécessite en soi quelques éclaircissements puisqu'il existe, en effet, une différence, selon que ce terme est utilisé dans les pays régis par la common law ou dans les pays de tradition romano-germanique.

\subsection{Les contrats dans la common law}

Comme le droit des contrats est éminemment jurisprudentiel dans la famille de la common law, il est difficile de trouver une définition unique du concept de contrat. Dans le droit anglais, par exemple, il n'y a pas une définition légale du contrat. Aux États-Unis, le American Restatement of the Law Second, Contracts (Volume 1) propose, plutôt qu'une définition, une affirmation qui nous sert de point de départ: «a promise or set of promises, for breach of which the law gives a remedy, or the performance of which the law in some way recognizes as a duty» (American Law Institute, 1981). De cette définition, nous pouvons déduire que, en général, dans la famille de la common law, l'élément le plus important du contrat est la promesse faite, tandis que pour le droit français ${ }^{1}$ et le droit espagnol, l'élément-clé est le consensus. De fait, le droit anglo-saxon insiste sur l'unique réalité sous-jacente aux contrats, à savoir la promesse en soi, alors que le droit français et le droit espagnol s'appuient sur un concept plus abstrait, celui du consensus (Samuel 2003: 69). Nous ne pouvons oublier que, dans le droit anglo-saxon, il n'existe pas d'obligation générale de bonne foi. La tromperie active n'est pas tolérée, mais l'obligation de communiquer à l'autre partie les informations qui pourraient lui être avantageuses n'existe pas: il incombe à chacune des parties de s'informer et de protéger ses intérêts, au point où certains auteurs ont proclamé l'existence même du principe de «trompe qui peut» (Caillé 2003: 33).

En général, la technique utilisée lors de la rédaction des contrats anglo-saxons diffère de celle qui est employée par les Français et les Espagnols (voir, pour une analyse plus détaillée, Borja [2000] et Alcaraz [2000]), notamment en raison de la volonté des rédacteurs de mettre par écrit absolument tout ce qui a été négocié. En effet, ils savent qu'en cas de désaccord, les tribunaux anglais auront recours à une interprétation très littérale, contrairement aux tribunaux français ou espagnols (Lefevbre 2003: 80). Alors que les juristes de la civil law ne voient aucun intérêt pratique à inclure, dans la rédaction du contrat, des clauses qui, de toute façon, sont considérées comme des lois supplétives, les juristes de la common law ont une perception selon laquelle «implied terms (even statutory implied terms) as a last resort, [have] to be avoided if at all possible» (Cartwright 2007: 193). Ceci est l'une des 
raisons pour lesquelles les contrats de la common law contiennent des clauses très longues, car ils ne se limitent pas à reprendre quelques concepts juridiques bien établis, mais cherchent à les préciser de manière explicite. Par exemple, l'exonération résultant d'un empêchement (cas de force majeure) se verra complétée par une énumération exhaustive des cas qui peuvent être considérés comme étant de force majeure. C'est dans ce contexte que doivent s'insérer les clauses de limitation ou d'exonération de responsabilité.

\subsection{Les clauses de limitation ou d'exonération de responsabilité}

Ces clauses comportent une énumération très exhaustive des dommages, car les parties veulent s'assurer que le seul dommage qui puisse être réparé est celui qui porte atteinte à l'intérêt recherché au moment de la conclusion du contrat, celui qui a justifié sa signature, c'est-à-dire le bénéfice du contrat (normal loss). Dès lors, on cite non seulement les différents types de dommages qui peuvent exister (special, indirect, incidental, consequential, punitive damages...), mais aussi les éléments qui constituent le dommage (lost revenues, profits, data).

Tout d'abord, il faut préciser que, autant en droit anglo-saxon qu'en droit espagnol et français, ces clauses sont considérées comme étant inapplicables quand le dommage est dû au dol ou à une faute grave. Par ailleurs, ce type de clause est souvent réputé abusif, et de ce fait, peut faire l'objet d'une annulation, raison pour laquelle les juristes prêtent une attention toute spéciale à leur libellé. Les tribunaux interprètent ces clauses de façon très restrictive et auront tendance, par exemple, à déclarer nulle l'exonération de responsabilité lorsqu'il s'agit d'un contrat passé par un professionnel avec un consommateur, car ce sont des dispositions contraires aux lois de protection des droits des consommateurs (voir l'article 10.1 de la loi espagnole de responsabilité civile du fait des produits défectueux).

Ainsi, la législation espagnole en la matière prescrit la nullité des clauses qui utilisent des termes obscurs:

Una cláusula puede ser oscura, por ser incierta en sus propios términos, al haber sido utilizadas expresiones anfibiológicas, con doble sentido o más de una acepción, de modo que no es posible conocer el alcance real de la cláusula sin proceder a una posterior concreción (Nieto 2000:343).

En droit espagnol, les clauses d'exonération absolue en matière de responsabilité, passées avec les consommateurs, sont considérées comme nulles. De plus, la règle de interpretatio contra estipulatorem (dans le monde anglo-saxon, on préfère utiliser l'expression contra preferentem) est appliquée, c'est-à-dire que l'ambiguïté ou le manque de clarté d'une clause ne peut favoriser en aucun cas la partie qui l'a rédigée (art. 6.2 de la LCGC, loi espagnole sur les conditions générales du contrat). Néanmoins, et alors qu'il s'agit de clauses qui, bien souvent, sont nulles, les auteurs recommandent toujours leur inclusion dans la rédaction des contrats (Ribas 2003: 118-119).

En France, le ministère responsable des questions liées au droit de la consommation a créé la Commission des clauses abusives, laquelle veille à l'application des normes de protection des consommateurs dans tout type de contrats. Cette commission a été créée par les articles 35 et suivants de la loi du 10 janvier 1978 et, depuis sa création, elle a émis plus de quarante recommandations, l'une d'entre elles portant précisément sur «les contrats proposés par les éditeurs ou distributeurs de logiciels 
ou progiciels destinés à l'utilisation sur micro-ordinateurs» (Commission des clauses abusives 1995: Recommandation 95-02). Il y est expressément stipulé l'élimination de ce type de clauses «les clauses qui ont pour objet ou pour effet: [...] $5^{\circ}$ D’exonérer le professionnel de toute responsabilité du fait des conséquences dommageables de l'utilisation des logiciels qu'il commercialise».

Ces nombreuses contraintes juridiques et le haut niveau de standardisation des libellés restreignent considérablement le travail du traducteur quant au choix de la terminologie. En effet, le client juriste prêtera une attention particulière au fait que la traduction soit la plus proche du texte original. Par conséquent, la traduction mot à mot finit par s'imposer. Par ailleurs, les termes appartenant au domaine du droit des dommages sont à la fois polysémiques et très imprécis, du fait que les juristes ne se sont pas mis d'accord sur la terminologie qu'il convient d'utiliser.

\section{3. Étude des termes relatifs aux dommages en anglais et en espagnol}

Suivant le principe proposé par Constantinesco (cité dans Sarcevic 2000: 240), «the best way to establish the constituent characteristics of concepts is to examine all the original sources of the law of the particular legal systems: legislation, doctrine, case law, and customary law»; c'est ainsi que nous ferons appel au droit comparé pour analyser les problèmes de terminologie dans le cadre du droit des dommages.

\subsection{Stratégies de traduction}

Lors de la sélection de la technique de traduction à appliquer, nous formulons des critères de priorité en fonction de l'existence ou non du concept dans la langue ou culture cible. La traduction des termes qui désignent des institutions ou des concepts spécifiques d'un système juridique donné posent des problèmes de traduction qui ont été suffisamment étudiés par de nombreux auteurs (voir Sarcevic 2000: 233). Selon Terral (2004: 883), nous considérons que toutes les techniques (la paraphrase, le substitut descriptif, l'emprunt ou l'équivalent fonctionnel) sont valables dès lors qu'elles servent à éviter de possibles problèmes de compréhension. C'est ainsi que toute traduction doit tenir compte de la finalité du texte traduit et du destinataire de la traduction.

\subsubsection{Le concept et les termes existent déjà dans la culture et la langue cibles}

Lorsque le concept existe déjà dans la langue ou la culture cible (l'espagnol, dans le cas présent) nous chercherons un équivalent, un terme qui remplisse la même fonction que celui de la culture de départ. C'est ainsi que Sarcevic (2000: 236) a repris une définition créée auparavant: "a functional equivalent is a term designating a concept or institution of the target legal system having the same function as a particular concept of the source legal system ».

Dans le tableau 1, nous présentons des exemples de termes anglais et des équivalents possibles qui sont habituellement utilisés dans les traductions de l'anglais vers l'espagnol. Nous avons appliqué les critères fixés par Sarcevic (2000: 241-249) pour déterminer le degré d'admissibilité des équivalents proposés. Nous avons signalé avec un astérisque ${ }^{*}$ ) les équivalents qui seraient incorrects dans le contexte de la traduction des contrats que nous analysons. Ce repérage nous permet de souligner que, 
pour quatre des douze termes analysés, la traduction lexicale ou étymologique ne serait pas acceptable, car elle pourrait donner lieu à une erreur de sens. Les équivalents en adéquation avec la tradition de la langue juridique espagnole sont marqués d'une croix $\left(^{+}\right)$.

TABLEAU 1

Termes juridiques relatifs à damages et leurs équivalents en espagnol

\begin{tabular}{|c|c|c|c|}
\hline Terme en anglais & $\begin{array}{l}\text { Équivalent } \\
\text { fonctionnel }\end{array}$ & $\begin{array}{c}\text { Équivalent lexical ou } \\
\text { étymologique }\end{array}$ & Autres équivalences \\
\hline $\begin{array}{l}\text { Contributory } \\
\text { negligence }\end{array}$ & $\begin{array}{l}{ }^{+} \text {Concurrencia de } \\
\text { culpas }\end{array}$ & $\begin{array}{l}\text { Negligencia } \\
\text { concurrente }\end{array}$ & $\begin{array}{l}\text { Imprudencia } \\
\text { negligente o } \\
\text { contribuyente } \\
\text { (Alcaraz et Hugues } \\
2001: 103 \text { ) }\end{array}$ \\
\hline Duty to mitigate & Principio de buena fe & ${ }^{+}$Deber de mitigar & $\begin{array}{l}{ }^{*} \text { Atenuación o } \\
\text { aminoración (Alcaraz } \\
\text { et Hugues 2001: 254) }\end{array}$ \\
\hline Damages & $\begin{array}{l}{ }^{*} \text { Indemnización de } \\
\text { daños y perjuicios }\end{array}$ & ${ }^{\star}$ Daños & $\begin{array}{l}\text { Derecho de } \\
\text { responsabilidad civil }\end{array}$ \\
\hline Liquidated damages & ${ }^{+}$Cláusula penal & Daños líquidos & ${ }^{+}$Cláusula estimatoria \\
\hline Punitive damages & $\begin{array}{l}\text { Daños no } \\
\text { compensatorios } \\
\text { Daños punitivos }\end{array}$ & ${ }^{+}$Daños punitivos & Daños ejemplares \\
\hline Nominal damages & $\begin{array}{l}\text { +Indemnización } \\
\text { simbólica }\end{array}$ & ${ }^{\star}$ Daños nominales & $\begin{array}{l}\text { Indemnización sin } \\
\text { daño }\end{array}$ \\
\hline Specific performance & $\begin{array}{l}\text { Ejecución en natura, } \\
\text { Ejecución forzosa, } \\
\text { +Reparación en natura, } \\
\text { Cumplimiento por } \\
\text { equivalente }\end{array}$ & $\begin{array}{l}\text { Cumplimiento } \\
\text { específico }\end{array}$ & $\begin{array}{l}\text { Cumplimiento de } \\
\text { obligaciones no } \\
\text { dinerarias }\end{array}$ \\
\hline Lost profits & ${ }^{+}$Lucro cesante & Ganancia perdida & $\begin{array}{l}\text { Ganancia o lucro o } \\
\text { frustrado }\end{array}$ \\
\hline Pecuniary damages & ${ }^{+}$Daños patrimoniales & Daños pecuniarios & \\
\hline Strict liability & $\begin{array}{l}{ }^{+} \text {Responsabilidad } \\
\text { objetiva }\end{array}$ & $\begin{array}{l}\text { Responsabilidad } \\
\text { estricta }\end{array}$ & $\begin{array}{l}{ }^{+} \text {Responsabilidad sin } \\
\text { culpa }\end{array}$ \\
\hline Fault & ${ }^{+} \mathrm{Culpa}$ & ${ }^{*}$ Falta & \\
\hline Implied terms & ${ }^{+}$Derecho supletorio & *Términos implícitos & Leyes supletorias \\
\hline
\end{tabular}

La traduction du terme damages par daño donne lieu à une erreur de traduction. En effet, la définition que donne McGregor (2003:3) du concept dénoté par damages est: «the pecuniary compensation, obtainable by success in an action, for a wrong which is either a tort or a breach of contract, the compensation being in the form of a lump sum awarded at one time, unconditionally and in sterling». Cette définition correspond en espagnol à celle de indemnización de daños y perjuicios et non pas au dommage en soi (daño).

Dans d'autres exemples, l'admissibilité d'un équivalent est déterminée par le contexte de traduction. Par exemple, les textes du droit international montrent une préférence pour l'utilisation de termes qui sont plus neutres et qui ne peuvent pas être confondus avec des termes existant dans la culture d'un pays donné. Ainsi, le terme specific performance est traduit en espagnol dans la Convention de Vienne (Convention des Nations Unies sur les contrats de vente internationaux de marchandises) par cumplimiento específico. De cette façon, le texte, dont la portée est internationale, 
vise à mettre en exergue le fait que cette action n'a pas le même poids spécifique dans les pays de la common law que dans ceux de la famille romano-germanique. Par conséquent, dans ce contexte, on ne donne pas préférence à des équivalents fonctionnels qui sont plus communément utilisés dans le contexte du droit espagnol, tels que ejecución en natura, ejecución forzosa, reparación en natura, cumplimiento por equivalente. Dans d'autres cas, l'équivalent fonctionnel permet une meilleure compréhension, car justement, il s'agit d'un terme plus naturel dans la langue d'arrivée, par exemple les termes cláusula penal (liquidated damages), lucro cesante (lost profits) ou bien daños patrimoniales (pecuniary damages). Dans le cas du terme fault, sa traduction par l'équivalent fonctionnel en espagnol culpa s'impose à fin d'éviter un contresens, car le terme espagnol falta aurait plutôt des connotations de droit pénal.

\subsubsection{Le cas de l'expression daños y perjuicios}

L'expression espagnole daños y perjuicios est figée. Même s'il est possible, d'un point de vue sémantique, de distinguer les concepts dénotés respectivement par daños et perjuicios, le fait est que les deux éléments ont chacun un sens unitaire vis-à-vis des effets juridiques qui en dérivent. Le Diccionario de Derecho en offre la définition suivante:

Es el menoscabo material o moral causado a una persona y del cual, por ser otra la responsable, habrá de responder ésta ante aquella. Aunque se habla habitualmente de daños y perjuicios de forma conjunta, cabe distinguirlos: son daños los menoscabos directos, mientras que los perjuicios son los menoscabos derivados de los daños (Ribó 1995: 296).

Par conséquent, si nous avons comme unique source de documentation ce dictionnaire juridique, nous arriverons à la conclusion que l'on appelle daños ce qui est infligé directement à l'objet du contrat et que l'on nomme perjuicios ce qui en découle. Nous pourrions même déduire que le terme daños correspond en fait au concept dénoté par direct damages et perjuicios, au concept dénoté par indirect damages. Tel n'est cependant pas l'usage que l'on fait de ces termes dans le domaine juridique.

La plupart du temps, la doctrine considère que la distinction entre daños et perjuicios a un sens purement académique, sans aucune portée juridique, et que les deux termes sont synonymes. Ainsi, Vázquez Sotelo, après avoir soutenu que pour être réparé, un dommage doit être direct, c'est-à-dire dériver immédiatement du fait dommageable, affirme ce qui suit:

La reparación del daño ha pretendido distinguirse de la indemnización del perjuicio tomando como base los textos legales. Pero en realidad esas dos expresiones no son más que un pleonasmo jurídico. Jurídicamente las dos expresiones significan lo mismo. Es inútil querer distinguir [...] la reparación y la indemnización a base de referir la primera al daño y la segunda al perjuicio. Considerando como "daño" el directamente causado y "perjuicio" el indirectamente recibido. Puede verse en los profundos comentarios de Gómez Orbaneja la refutación del ejemplo que proponía Escriche quien decía que en el caso del pedrisco, causa mucho daño a las cosechas, mientras que la disminución del precio del trigo causa mucho perjuicio al cultivador ("sospechamos que dicho escritor no era un jurista”). Daños y perjuicios, reparación e indemnización son conceptos absolutamente idénticos (Vázquez Sotelo 1994: 134). 
Cet auteur signale que la jurisprudence n'a pas de position clairement définie, c'est pourquoi certains auteurs ont voulu identifier le concept correspondant à daños avec le damnum emergens et celui correspondant à perjuicios avec le concept dénoté par lucro cesante ou ont encore considéré le concept dénoté par daño comme une modalité du concept dénoté par perjuicio. Néanmoins, cette distinction, qui n’a pas une grande importance théorique, n'a aucune répercussion dans la pratique, car la jurisprudence espagnole a toujours appliqué les deux concepts d'une façon unitaire.

La contextualisation de l'expression daños y perjuicios nous permet de confirmer que la différence sémantique entre les deux termes ne correspond pas à la distinction académique que la doctrine établit entre daños directos et daños indirectos. Une fois la doctrine analysée, nous pouvons donc affirmer que l'équivalent fonctionnel de indemnización de daños y perjuicios est, en anglais, damages. En effet, en anglais, comme nous l'avons vu dans la définition de McGregor (2003: 3), le concept correspondant fait référence à la compensation économique qui est octroyée lorsqu’il $\mathrm{y}$ a un dommage et non pas au dommage en soi.

Dans le langage juridique, nombreux sont les exemples de termes qui peuvent être distingués sur le plan sémantique, mais auxquels le droit n'attribue pas de conséquences juridiques différentes. À titre d'exemple, on peut citer la différence entre caso fortuito et fuerza mayor. Différents auteurs ont clairement cerné ces deux termes (Mazeaud et Tunc, cités dans Cuevillas 2000: 175), définissant caso fortuito comme celui qui est le fruit du hasard, de la chance, de l'aléatoire, ce qui survient de façon inattendue..., tandis que fuerza mayor est un terme qui présuppose une force étrangère qui empêche l'action volontaire. Néanmoins, l'impact juridique est nul, à tel point que Jordano (1987: 216) en arrive à qualifier la question de «diversión académica». Du point de vue de la science de la traduction, par contre, cette différence n'est pas un problème pour le traducteur qui peut trouver des équivalents fonctionnels en anglais: caso fortuito peut se traduire par Act of God et fuerza mayor par force $\underline{\text { majeure }}^{2}$, en plus de pouvoir avoir recours à des paraphrases largement utilisées tant par UNIDROIT que par les Principes européens du droit des contrats (voir aussi Vicente 2002).

Le Código civil español nous fournit d'autres exemples d'expressions dont la traduction littérale peut entraîner des erreurs de sens, en particulier dans les situations de rétrotraduction vers l'anglais. Par ailleurs, utiliser l'expression resarcimiento de daños y abono de intereses (articles 1123-2, 1147-2 et 1152-1 du Código civil espa$\tilde{n} o l$ ), n'implique pas que la réparation doit, de manière automatique, inclure aussi bien les dommages que les intérêts. Il s'agirait plutôt d'un calque de l'expression française dommages et intérêts. Cette expression est utilisée dans de nombreux arrêts de la cour de cassation espagnole (Tribunal Supremo), compilés par Clemente (1998: 556) et dont l'analyse, sur le plan juridique, permet d'affirmer que l'expression resarcimiento de daños y abono de intereses, telle qu'elle apparaît dans le Código civil español, a le même sens que indemnización de daños y perjuicios. Ainsi, le Tribunal Supremo a établi que:

el abono de intereses a que se alude en el artículo 1124-2, no se refiere a la suma dineraria en concepto de fruto civil por disponer de dinero ajeno y como compensación por su disposición y disfrute, sino que la expresión "resarcimiento de daños y...[sic]" contenida en el citado precepto legal, es sinónima de la indemnización de daños y 
perjuicios, refiriéndose el segundo concepto más bien a ganancias dejadas de obtener (Tribunal Supremo, STS de 10 de octubre de 1980³).

Dès lors, dans ce contexte, l'équivalent fonctionnel de indemnización de daños y abono de intereses sera, en anglais, uniquement le terme damages. Il faut, en plus, savoir qu'en droit espagnol, l'indemnisation des dommages ne comporte pas de façon automatique le paiement d'intérêts.

\subsubsection{Les concepts des cultures source et cible ne correspondent que partiellement}

Selon Sarcevic,

while some functional equivalents are always acceptable (near equivalence) or never acceptable (non-equivalence), most functional equivalents fall into the category of partial equivalence. [...] In such cases, the acceptability of a functional equivalent usally depends on context (Sarcevic 2000: 241).

C'est ainsi que Santamaria (2003: 30) définit ces équivalents comme étant des «équivalents contextuels ». Il s'agit là de cas où la recherche de l'équivalent passe par une analyse contextuelle pour aboutir à une équivalence qui ne sera que partielle.

À titre d'exemple, nous pouvons citer le concept correspondant aux termes marital property ou community property. Il s'agit du régime matrimonial en vertu duquel les conjoints forment une communauté réduite aux acquêts; ce système s'oppose au régime dit de separate property. Dans un contexte de traduction-document, nous pourrions avoir recours à l'équivalent fonctionnel en vigueur dans le droit espagnol, à savoir, sociedad de gananciales (régi par les articles 1344 et suivants du Código civil español). Cependant, dans un contexte de traduction-instrument, dans lequel le concept doit s'appliquer en Espagne, de par l'application des normes du droit international privé, traduire community property par sociedad de gananciales impliquerait une erreur de sens grave, car les effets n'en sont pas équivalents. En effet, en droit espagnol, lorsque l'un des époux décède, on procède à la dissolution de ladite société et au partage des biens entre le conjoint survivant (qui en reçoit la moitié) et les héritiers du défunt. En droit anglo-saxon, il existe un ius accrescendi de la partie des biens qui formaient la propriété commune des deux conjoints (community property: tout le mobilier, les bijoux, etc., ainsi que le foyer conjugal) et qui de ce fait devient la seule propriété du conjoint survivant. Cette situation, qui peut se produire, par exemple, lorsqu'un juge espagnol est l'autorité compétente pour exécuter le testament d'un citoyen anglais. La meilleure option pour le traducteur sera alors de chercher un hyperonyme qui ne mette pas directement en cause les effets légaux de l'institution: Alcaraz et Hugues (2001: 92) reprennent, par exemple, le terme sociedad conyugal; une autre option, lorsque cela est possible, pourrait être d'inclure une note explicative en bas de page ${ }^{4}$.

Pour ce qui est du domaine de la responsabilité civile, nous trouvons l'expression vicarious liability qui, en droit anglo-saxon, est utilisée pour définir la responsabilité indirecte qui incombe au défendeur, face aux tiers, lorsque celui-ci a le droit et la capacité de contrôle, pour autant qu'il retire un intérêt économique direct des activités illégales (Clemente et Cavanillas 2003 : 47). En droit espagnol, nous parlerions, dans ce cas, de responsabilidad por hechos ajenos o por daños de terceros - responsabilité du fait d'autrui en droit français - tel qu'il est prévu dans l'article 1903 
du Código civil español. Dans ce sens, nous pourrions affirmer que l'équivalent fonctionnel de vicarious liability est responsabilidad por daños de terceros ou responsabilidad por hecho ajeno (tels qu'ils sont employés, par exemple, dans Salvador Coderch 2002: 16). Néanmoins, Roca (2003: 111) préfère, pour sa part, transposer l'institution anglo-saxonne et nomme responsabilidad vicaria l'action directe qui s'exerce sur les entrepreneurs afin de la distinguer de la responsabilité des parents et des éducateurs. Ainsi, nous nous trouvons face à une institution, vicarious liability, qui n'existe pas en droit positif espagnol et pour laquelle certains auteurs (Roca 2003: 111) ont choisi de réaliser une transposition linguistique à travers le calque, tandis que d'autres (Salvador Coderch 2002: 16) préfèrent utiliser un équivalent partiel qui suppose un hyperonyme.

\subsubsection{Le cas de special, indirect, consequential, incidental and punitive damages}

Cette énumération correspond à des catégories typiques de la common law, développées tant par la jurisprudence que par la voie législative et doctrinale. La jurisprudence anglo-saxonne a créé les classifications qui distinguent general and special damages ainsi que normal and consequential losses, catégories qui ont été élaborées par la doctrine à partir de l'emblématique affaire Hadley v. Baxendale. Incidental damages dénote un concept dont nous trouvons la définition dans l'Uniform Commercial Code (UCC), de même que consequential damages. Quant à indirect damages, nous ne l'avons trouvé qu'au sein de l'expression indirect or consequential damages; même si quelques lois spécifiques utilisent cette terminologie, son usage autonome ne semble pas évident. Il s'agit de termes avec un contenu conceptuel variable et incertain, étant donné qu'ils ne s'interprètent pas de la même façon et varient en fonction du contexte dans lequel ils apparaissent. Concrètement, consequential damages acquiert un sens beaucoup plus restreint dans des clauses d'exonération ou de limitation de responsabilité (McGregor 2003: 26-27). Ces catégories s'utilisent de façon accrue dans le domaine des relations commerciales internationales. Pourtant, les auteurs des textes normatifs internationaux, conscients du fait qu'il s'agit de figures spécifiques à la common law, les évitent et celles-ci n’apparaissent ni dans les principes UNIDROIT, ni dans la Convention de Vienne, ni dans les Principes européens du droit des contrats (PEDC). En effet, les PEDC ont pour vocation d'éviter, dans la mesure du possible, les termes qui peuvent donner lieu à des interprétations divergentes dans les droits nationaux respectifs. Pour ce faire, «no se diferencia entre daños directos, indirectos, mediatos, inmediatos, etc.» (Navas 2000: 1247). Nous analysons plus en détail chacun des termes repérés dans ce qui suit.

En droit anglo-saxon, le concept correspondant à special damages concerne autant les circonstances particulières relatives la partie qui subit le préjudice que des questions de procédure.

L'examen d'ouvrages lexicographiques semble indiquer que indirect damages est un synonyme de consequential damages. Cette conclusion coïncide avec l'interprétation restrictive du terme, dans le contexte des clauses d'exonération de responsabilité, par la jurisprudence anglo-saxonne, où certains juges ont affirmé que consequential est synonyme de not direct.

D'autre part, le terme indirect damages s'utilise fréquemment dans certaines lois, comme celles qui concernent la navigation aérienne ou l'immobilier et la construction ${ }^{5}$. 
Dans ce contexte, elles acquièrent la signification de dommages purement économiques, parfois également nommés residual or stigmatic damages.

De même, le concept dénoté par indirect damages pourrait couvrir les dommages aux personnes ou aux biens étrangers à l'objet du contrat. Il s'agirait de ce que la doctrine nomme other losses, auquel cas ce serait une partie de la définition de consequential losses reprise tant dans l'UCC que dans le Uniform Computer Information Transactions Act (UCITA) ${ }^{6}$. Dès lors, l'idée dénotée par indirect serait incluse dans celle de consequential.

Nous ne nous étendrons pas ici sur l'analyse des différents sens que consequential damages acquiert en droit anglo-saxon ${ }^{7}$, étant donné que nous nous concentrerons sur la traduction de la clause précédemment citée. Ainsi, du fait qu'il est polysémique, nous devons avoir recours à sa contextualisation afin de chercher le sens le plus proche. Les lois particulières lui ont octroyé une définition spécifique et c'est à elles que nous aurons recours afin de comprendre son sens. Dans ce cas, nous pensons que la source qui s'approche le plus du domaine des contrats de licence d'utilisation des programmes d'ordinateur est la législation des États-Unis en la matière (UCITA), qui définit ce type de dommage comme:

Any loss resulting from general or particular requirements and needs of which the breaching party at the time of contracting had reason to know and which could not reasonably be prevented and (ii) any injury to an individual or damage to property other than the subject matter of the transaction proximately resulting from breach of warranty.

Le concept dénoté par incidental damages fait référence à ces dépenses qui résultent de la non-exécution ainsi qu'à celles qui sont nécessaires pour réaliser un marché de couverture.

Dans le cas de punitive damages, le concept correspond à une indemnisation qui ne cherche pas à compenser les dommages, mais bien à punir, c'est-à-dire à imposer une peine. À notre avis, cette notion trouve son expression dans le terme espagnol daños punitivos; en aucun cas. il ne s'agirait d'une clause pénale du droit espagnol, qui ressemble plus, dans le droit anglo-saxon, à la notion dénotée par liquidated damages.

En dernier lieu, mais de façon tout aussi importante, l'analyse du droit privé espagnol nous permet de constater que ni le Código civil español ni les lois spéciales n'incorporent ces définitions. La doctrine connaît des classifications similaires telles que la distinction entre daños directos et daños indirectos (Vicente 2002: 73) ou entre daños mediatos et daños inmediatos (Navas 2000: 1255), mais nous ne pouvons affirmer qu'il s'agit de termes équivalents. Par contre, nombreux sont les formulaires et les auteurs qui utilisent des expressions telles que daños consecuentes (Carrasco ${ }^{8}$ 2000: 716; Llaneza 2004: 83), daños consecutivos (Salelles 2007: 220) ou daños consecuenciales (Carrasco 2000: 740; Gómez Pomar et Ruiz García 2002: 6).

Quant au terme consequential damages, il est particulièrement compliqué d'établir un équivalent puisqu'il s'agit d'un terme polysémique. C’est pour ce motif que nous avons élaboré la figure suivante, dans laquelle on peut observer les différents sens de ce terme. 
FIGURE 1

Analyse du terme consequential damages et de ses possibles équivalents en espagnol

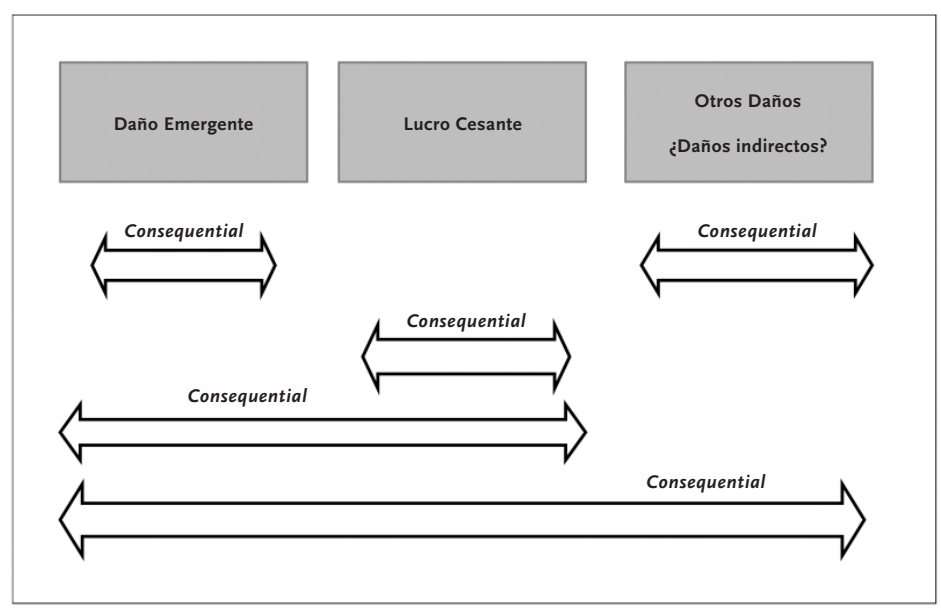

Grâce à l'étude de la doctrine et de divers corpus, les traductions suivantes de consequential damages ont été identifiées:

- daños consecuenciales : équivalent mot à mot

- daños consecutivos : calque lexical

- daños consecuentes: calque lexical

- daños secundarios : équivalent contextuel

Dans la doctrine française, Tallon, représentant français auprès de la Commission Lando qui a supervisé les travaux de traduction des PEDC et qui a aussi participé à la rédaction des principes de UNIDROIT, se prononce de façon catégorique sur cette question: "consequential damages, as well as incidental damages are very difficult expression to translate into French since the expression "préjudice indirect" bears a quite different meaning» (Tallon 1992: 682). De même dans la doctrine italienne, Draetta $(1991$ : 491) considère que les notions correspondant à consequential damages et à indirect damages "could not be more different». Nous devons également mentionner l'étude que Fofaria (2006) a réalisée sur le concept dénoté par consequential and indirect losses. Cet auteur souligne que les effets juridiques de l'exclusion de ce type de dommages dans le droit anglo-saxon sont très différents de ceux qui se produisent dans le droit français et que, dès lors, la transposition juridique de ces institutions donne lieu à un résultat non voulu par les parties.

En droit espagnol, la doctrine n'analyse habituellement pas ces catégories. De fait, l'on peut affirmer, presque catégoriquement, que les auteurs spécialisés en droit civil les ignorent et n'y font référence que lorsqu'ils analysent le droit comparé. Parmi les auteurs qui essayent d'étudier ce concept, le seul que nous ayons trouvé qui réalise une étude en profondeur des différentes significations que peut avoir ce type de classifications des dommages est un spécialiste en droit commercial (Salelles 2007: 225). Il semble, dès lors, que c'est dans le domaine du droit commercial que l'on trouve le plus de perméabilité à ce type de catégories, en dépit du fait qu'une grande partie du corpus électronique qui se trouve sur Internet et qui inclut ces termes dans des contrats en 
espagnol relèverait directement du droit civil, étant donné qu'il s'agit de contrats passés avec des consommateurs. Par conséquent, même si, dans une situation hypothétique, l'on considérait comme étant correcte la transposition linguistique de ces catégories dans le domaine du droit commercial, on pourrait soutenir qu'il ne s'agit pas d'un équivalent acceptable dans le cadre d'un contrat régi par le droit civil.

Dès lors, le travail de documentation du traducteur dans ce domaine mène, en quelque sorte, à une impasse, liée, d'une part, à l'équivalence insuffisante des termes trouvés et, d'autre part, à la grande spécificité du domaine auquel ces termes appartiennent, à savoir des clauses qui impliquent l'exonération de montants extrêmement élevés à titre d'indemnisation de dommages et intérêts. Enfin, il ne faut pas non plus oublier l'effet de la pression des juristes qui ont pour habitude de dire «traduisez mot à mot et laissez le travail d'interprétation aux juristes». C'est pourquoi nous aborderons l'analyse traductologique de cette expression en nous basant sur le critère que nous avons retenu pour choisir la méthode de traduction: le droit applicable au contrat.

\subsection{Propositions de traduction}

\subsubsection{Première proposition}

Si la clause se trouve insérée dans un contrat régi par le droit anglo-saxon ${ }^{9}$ (soit parce qu'elle résulte d'une clause de soumission expresse à ce droit, soit parce que celui-ci est applicable en vertu des normes sur les conflits de lois), nous procéderons à une traduction référentielle ou informative, c'est-à-dire une traduction-document. Dans ce cas, nous nous limiterons à vérifier si la doctrine espagnole a transposé ces concepts et, dès lors, si ces notions existent en espagnol. Après avoir vérifié que divers auteurs les utilisent (Carrasco 2000; Salelles 2007), nous effectuerons une transposition linguistique et nous traduirons de la façon suivante.

TABLEAU 2

Équivalents des termes spécifiques de damages dans le cadre d'une traduction-document

\begin{tabular}{|c|c|}
\hline Terme anglais & Transposition linguistique \\
\hline special damages & daños especiales \\
\hline indirect damages & daños indirectos \\
\hline $\begin{array}{l}\text { consequential } \\
\underline{\text { damages }}\end{array}$ & $\begin{array}{l}\text { - daños consecuentes }{ }^{10} \text { : que siguen en orden respecto de algo (Carrasco } \\
\text { 2000; Llaneza 2004). } \\
\text { - daños consecuenciales: calco del inglés, no aceptado por la RAE } \\
\text { (Gómez Pomar et Ruiz García } 2002 \text {; Carrasco } 2000 \text { y numerosos corpus } \\
\text { en Internet). } \\
\text { - daños consecutivos: que siguen inmediatamente a otra o es } \\
\text { consecuencia de ella, (Salelles } 2007 \text {, des auteurs français qui utilisent } \\
\text { dommages consecutifs). } \\
\text { - daños secundarios: las formas anteriores tienen la ventaja de que } \\
\text { fonéticamente se asemejan al término en inglés, mientras que este } \\
\text { término refleja el sentido de consecuencia indirecta y no principal. }\end{array}$ \\
\hline$\frac{\text { incidental }}{\text { damages }}$ & daños incidentales: en el sentido de hecho sobrevenido ${ }^{11}$ \\
\hline punitive damages & daños punitivos: que tienen intención de penalizar \\
\hline
\end{tabular}


Ce type de traduction, qui correspond presque à une traduction mot à mot, ne prétend pas transposer des concepts juridiques, mais uniquement informer sur un droit non national avec des termes qui appartiennent à la langue générale et au domaine du droit. Le lecteur, s'il est spécialisé en droit comparé, saura bien où trouver les sources pour se documenter et, s'il n'a pas cette formation, il devra faire appel à un juriste anglo-saxon afin qu'il l'assiste. Dans ce cas, la fonction du traducteur est uniquement de communiquer une réalité étrangère et de s'assurer que ces termes existent en espagnol, même s'il s'agit de transferts culturels réalisés par la doctrine propre du droit comparé. De plus, le fait d'avoir vérifié la transposition linguistique permet au lecteur de ne pas se tromper et d'éviter qu'ils ne les prennent pour des institutions du droit espagnol.

\subsubsection{Deuxième proposition}

Dans le cas où le contrat est régi par le droit espagnol, la fonction de la traduction doit varier, étant donné que les instruments d'interprétation ne seront pas les notions recueillies par la doctrine, mais bien le droit positif espagnol. Dans ce cas, la traduction mot à mot nous mènerait peut-être à des résultats incongrus, étant donné qu'il s'agit d'institutions qui n'existent pas en droit espagnol et qui ne reprennent pas non plus, de façon expresse, les instruments internationaux en la matière. Dès lors, si nous voulons que les juges espagnols concèdent à cette clause les mêmes effets qu'elle produit dans le droit anglo-saxon, nous devrons paraphraser ces termes. La difficulté de la traduction réside donc dans l'explication de ces concepts sans pour autant en éliminer l'ambigüité des termes du texte de départ.

TABLEAU 3

Paraphrases en espagnol des termes spécifiques de damages

\begin{tabular}{|c|c|}
\hline Terme & Paraphrases proposées en espagnol \\
\hline special damages & $\begin{array}{l}\text { - daños especiales debidos a las circunstancias particulares del cliente } \\
\text { - daños especialmente previstos por las partes }\end{array}$ \\
\hline indirect damages & $\begin{array}{l}\text { - daños indirectos o remotos } \\
\text { - daños no directos } \\
\text { - daños puramente económicos, daños residuales } \\
\text { - daños colaterales }\end{array}$ \\
\hline $\begin{array}{l}\text { consequential } \\
\text { damages }\end{array}$ & $\begin{array}{l}\text { - daños consecuentes que resulten de cualquier pérdida producida por } \\
\text { circunstancias generales o particulares, que la parte incumplidora hubiera } \\
\text { podido conocer en el momento de celebrar el contrato y que no haya } \\
\text { podido evitar de forma razonable así como cualquier otro tipo de daño a } \\
\text { personas o bienes producido por la falta de garantía. }\end{array}$ \\
\hline $\begin{array}{l}\text { incidental } \\
\text { damages }\end{array}$ & - daños emergentes \\
\hline $\begin{array}{l}\text { punitives } \\
\text { damages }\end{array}$ & - daños punitivos \\
\hline
\end{tabular}

Nous sommes conscients qu'une demande de traduction réelle n'admettrait pas des paraphrases telles que celles que nous proposons pour cerner le sens des termes à traduire. C'est la raison pour laquelle nous considérons que dans un contexte de traduction où le contrat serait soumis au droit espagnol, la solution doit passer par 
une traduction juridique de telle façon que les termes étudiés ici devraient être substitués par des expressions plus en accord avec le droit espagnol.

Certaines entreprises ont commencé traduire les clauses de limitation ou d'exclusion de responsabilité en fonction du droit applicable au contrat. C'est ainsi que nous pouvons trouver certaines versions en espagnol des contrats de licence de logiciels ou de systèmes d'exploitation qui réalisent une traduction juridique, c'est-à-dire donc plus orientée vers la langue d’arrivée. Nous présentons un exemple extrait du contrat de Microsoft pour son système d'exploitation Windows XP Professional, souscrit par l'Université Autonome de Barcelone en octobre 2002.

TABLEAU 4

Exemple d'adaptation, au droit espagnol, d'une clause d'exonération de responsabilité

\begin{tabular}{|l|l|}
\hline \multicolumn{1}{|c|}{ Version en anglais } & \multicolumn{1}{c|}{ Version en espagnol } \\
\hline $\begin{array}{l}\text { Exclusion of incidental, consequential and } \\
\text { certain other damages. To the maximum } \\
\text { extent permitted by applicable law, in no } \\
\text { event shall Microsoft or its suppliers be liable } \\
\text { for any special, incidental, punitive, indirect, } \\
\text { or consequential damages whatsoever }[\ldots]\end{array}$ & $\begin{array}{l}\text { [...] Microsoft y sus proveedores no serán } \\
\text { responsables por los daños de cualquier } \\
\text { naturaleza, salvo por dolo o negligencia grave, } \\
\text { incluyendo, entre otros, los daños por lucro } \\
\text { comercial cesante, interrupción de negocio, } \\
\text { pérdida de información comercial u otra } \\
\text { pérdida económica [...] }\end{array}$ \\
\hline
\end{tabular}

La recherche d'équivalents possibles pour procéder à la traduction des termes juridiques doit ainsi passer par une analyse comparative de la branche de droit dans laquelle ils s'insèrent. Cela nous permettrait d'établir le degré d'équivalence et, dans un deuxième temps, de procéder à la traduction en appliquant une approche fonctionnaliste qui nous permette de vérifier si les décisions prises en matière de traduction correspondent aux situations de communication où peut se trouver ce type de clauses. En somme, la traduction juridique ne peut se limiter à une approche juridique et linguistique, mais doit, en outre, examiner toutes les considérations pragmatiques qui entourent l'opération de la traduction telles que les facteurs communicatifs et culturels.

\section{Sources documentaires juridiques}

Comme nous l'avons vu jusqu'à présent, la traduction juridique doit se fonder sur un travail exhaustif de documentation de la part du traducteur. Les dictionnaires ne suffisent pas à résoudre les problèmes conceptuels, surtout quand les termes sont culturellement marqués. D’autre part, si nous nous limitions à la simple recherche de textes parallèles, nous n'obtiendrions pour seul résultat que celui de perpétuer les pratiques de traduction, qui en aucun cas, n’ont été juridiquement vérifiées. C'est pour cela qu'une documentation correcte doit passer par une recherche dans les sources juridiques telles que la loi, la jurisprudence et la doctrine.

À titre d'exemple, dans les tableaux présentés en annexe, nous incluons quelques sources avec lesquelles nous avons travaillé et qui sont d'origine tant législative nationale (Código civil español, UCC, UCITA), internationale (CISG), jurisprudentielle (Hadley v. Baxendale, Maranto v. Goodyear Tire \& Rubber Co.) que lexicographique, sous la forme de dictionnaires unilingues spécialisés (Ribó 1995; Black's - Garner 2004) et bilingues (Alcaraz et Hugues 2001; Cabanellas et Hoague 1993). 


\section{Conclusion}

En résumé, un même terme du texte original peut se traduire par divers équivalents distincts selon la finalité poursuivie par le texte d'arrivée, qu'elle soit informative ou instrumentale (Nord 1997). Notre exemple de clause d'exonération en serait une bonne illustration, où les termes tels que daños especiales, indirectos, consecuentes, incidentales, punitivos, peuvent être traduits d'une certaine façon dans le cas d'une traduction de type informatif. En effet, dans ce cas, nous pouvons utiliser les emprunts des institutions juridiques étrangères étant donné que, comme nous l'avons vérifié, elles ont été transposées en droit espagnol par la doctrine. Par conséquent, l'acte de communication se produit sans distorsion. Cependant, lorsque la traduction est instrumentale, nous devons avoir recours à d'autres techniques de traduction qui respectent le droit positif national et qui, certainement, impliqueront un degré d'adaptation linguistique et juridique accru.

Quoi qu'il en soit, tant la traduction référentielle que la traduction instrumentale doivent être précédées d'un travail de documentation exhaustif. Ce travail de recherche documentaire peut être simplifié dans le premier cas, grâce à l'existence d'une doctrine abondante qui a incorporé ou transplanté par ses études les termes juridiques en anglais. Toutefois, il ne saurait être résolu par le seul recours aux moyens habituels, telle la consultation de dictionnaires spécialisés ou de textes parallèles. Le travail comparé est essentiel en matière de traduction juridique et les traducteurs apprécieraient que les juristes s'occupent davantage du droit comparé. Les exigences d'une mondialisation croissante et la réalité du travail quotidien du traducteur juridique, qui ne jouit toujours pas d'une reconnaissance sociale et économique suffisante, justifient une plus grande coopération entre juristes et traducteurs, ce qui favoriserait, en outre, la spécialisation de nouveaux professionnels dans des domaines multidisciplinaires, tels que le droit comparé et la linguistique juridique.

\section{NOTES}

1. L'article 1101 du Code civil français prévoit que: «Le contrat est une convention par laquelle une ou plusieurs personnes s'obligent, envers une ou plusieurs autres, à donner, à faire ou à ne pas faire quelque chose».

2. Le Black's Law Dictionary (Garner 2004: 674) reprend le terme force-majeure clause comme: «a contractual provision allocating the risk if performance becomes impossible or impracticable».

3. Tribunal Supremo, STS de 10 de octubre de 1980, RJ 1980/3619.

4. Nous considérons que le terme sociedad conyugal est un hyperonyme de sociedad de gananciales et que, dès lors, il pourrait être utilisé comme équivalent de l'institution anglo-saxonne. Cependant, comme le droit de la famille varie en fonction de son application territoriale et que, par exemple, en Navarre et dans plusieurs pays d'Amérique latine l'expression est utilisée comme équivalent de sociedad de gananciales, nous recommandons, là où cela est possible, d'ajouter une note du traducteur qui précise qu'il s'agit d'une équivalence partielle ou contextuelle et que les effets juridiques sont différents dans ces deux institutions.

5. À titre d'exemple, nous pouvons citer le New York Navigation Law de 1941 (art. 181-2) et la Comprehensive Environmental Response Compensation and Liability Act de 1980.

6. En fin de compte, cette loi n'a pas été incorporée au Uniform Commercial Code (UCC) puisque l'American Law Institute s'est retiré du projet, car il était en désaccord avec le texte. La National Conference of Commissioners on Uniform State Laws a fini par élaborer, seule, le texte définitif qui a été adopté en 1999.

7. Voir, à ce propos McGregor (2003: 25-27) et, pour une esquisse, la figure 1 .

8. Cet auteur utilise davantage consecuencial, sauf dans un cas, où il utilise consecuente. 
9. Cependant, d'un point de vue strictement juridique, on ne peut jamais affirmer catégoriquement qu'un autre droit national ne se verra pas appliqué. S’agissant de normes qui concernent la responsabilité civile, il serait suffisant qu'il se produise des dommages extracontractuels pour que l'on puisse échapper à un droit national plus restrictif.

10. Une recherche menée à l'aide du moteur de recherche Google (recherche en date du 3 mai 2010) pour les expressions suivantes a donné ce qui suit (nous avons associé les différents adjectifs recherchés avec indirecto afin de réduire au maximum les occurrences existant dans des contextes autres que ceux des énumérations de types de dommages). Pour «indirecto o consecuencial»: 539 pages web d'Espagne et 68700 pour tout Internet; «indirecto o consecutivo » : 2 pages web d'Espagne et 29 pour tout Internet; «indirecto o consecuente» : 29800 pages web d'Espagne et 52900 pour tout Internet: "secundario o indirecto » : 2480 pages web d'Espagne et 15600 pour tout Internet. L'utilisation du calque de l'anglais, c'est-à-dire consecuencial, est beaucoup plus répandu que les autres possibilités dans l'ensemble d'Internet, mais nous pouvons constater qu'en Espagne un nombre significatif de pages font usage de consecuente.

11. Ladjectif incidental est déjà utilisé dans le Código civil español avec le sens de «survenu» alors qu'à l'article 1270 il fait référence au dol accidentel qui n'oblige celui qui l'a employé qu'à payer des dommages et intérêts.

\section{RÉFÉRENCES}

Alcaraz, Enrique (2000): El Inglés Jurídico. Barcelona: Ariel.

Alcaraz, Enrique et Hugues, Brian (2001): Diccionario de Términos Jurídicos. Barcelona: Ariel.

AMERICAN LAW INSTITUTE (1981): American Restatement of the Law Second, Contracts, Vol. 1. États Unis: West.

Borja, Anabel (2000): El texto jurídico inglés y su traducción al español. Barcelona: Ariel.

Cabanellas, Guillermo et Hoague, Eleanor (1993): Diccionario Jurídico. Buenos Aires: Heliasta.

CAIlle, Alain (2003): De l'idée de contrat. Le contrat comme don à l'envers (et réciproquement). In: Christophe JAMIN et Denis MAzEAud, dir. La nouvelle crise du contrat. Paris: Dalloz, 27-50.

Carrasco, Angel (2000): Comentario al artículo 1.106. In: Manuel Albaladejo, dir. Comentarios al Código Civil y Compilaciones Forales. Barcelona: Bosch, 666-719.

CArtwright, John (2007): An Introduction to the English Law of Control for the Civil Lawyer. Oxford: Hart.

Clemente, Mario (1998): La facultad de resolver los contratos por incumplimiento. Valencia: Tirant Lo Blanch.

Clemente, Mario et Cavanillas, Santiago (2003): Responsabilidad civily contratos en internet. Granada: Comares.

CÓDIGO CIVIL ESPAÑOL, Real Decreto de 24 de julio de 1889. Consulté le $1^{\text {er }}$ mai 2010, <http://civil.udg.es/normacivil/estatal/CC/INDEXCC.htm>.

Cuevillas, Ignacio (2000): La relación de causalidad en la órbita del derecho de daños. Valencia: Tirant lo Blanch.

Dullion, Valerie (2007): Traduire les lois. Un éclairage culturel. La traduction en français des codes civils allemand et suisse autour de 1900. Cortil-Wodon: E.M.E. Éditions Modulaires Européennes.

FOFARIA, Akshai (2006): Excluding the recovery of "consequential and indirect losses" in English and French laws. Revue de Droit des Affaires Internationales. 5:597-615.

Garner, Bryan, éd. (2004) : Black's Law Dictionary. St Paul: Thomson-West.

Gómez Pomar, Fernando et Ruiz García, José Antonio (2002) : La noción del daño puramente económico: una visión crítica desde el análisis económico del derecho. InDret. 4:1-30. Consultée le 29 juin 2009, <http://www.indret.com/pdf/102_es.pdf>.

JoRDANo, Francisco (1987): La responsabilidad contractual. Madrid: Civitas.

Llaneza, Paloma (2004): E-contratos, modelos de contratos, cláusulas y condiciones generales comentadas. Barcelona: Bosch. 
McGregor, Harvey (2003): McGregor on Damages. Londres: Sweet \& Maxwell.

Mazeaud, Henri et Léon et Tunc, André (1963): Tratado teórico y práctico de la responsabilidad civil, delictual y contractual. $5^{e}$ ed. trad. por Luis Alcalá-Zamora. Buenos Aires: Ediciones Jurídicas Europa-América.

Navas, Susana (2000): El resarcimiento de daños en los Principios europeos de Derecho de los contratos (con especial referencia al Derecho español). Actualidad civil. 34:1235-1262.

Nieto, Ubaldo, dir. (2000): Condiciones generales de la contratación y cláusulas abusivas. Valladolid: Lex Nova.

Nord, Christiane (1997): Translating as a Purposeful Activity. Functionalist Approaches Explained. Manchester: St. Jerome.

Ribas, Javier (2003): Aspectos jurídicos del comercio electrónico en Internet. Cízur-Menor: Thomson-Aranzadi.

Ribó, Luis (1995): Diccionario de Derecho. Barcelona: Bosch.

Roca, Encarna (2003): Derecho de daños. Textos y materiales. Valencia: Tirant lo Blanch.

SALELLES, José Ramón (2007): Exclusión y limitación de responsabilidad en la contratación entre empresarios. Bolonia: Publicaciones del Real Colegio de España.

Salvador Coderch, Pablo (2002): Causalidad y responsabilidad. InDret. 1(2):1-14. Consulté le 29 juin 2009, <http://www.indret.com/pdf/008_es.pdf >.

Samuel, Geoffrey (2003): 'Réalisme' et 'efficacité' du droit anglais des contrats. In: Christophe Jamin et Denis Mazeaud, dir. La nouvelle crise du contrat. Paris: Dalloz, 69-81.

SAntamaria, Laura (2003): Eines multidisciplinàries per a l'ensenyament de la traducció angles-català: La traducció dels contractes. Revista de Llengua i Dret. 39:11-33.

SARCEvic, Susan (2000): New Approach to Legal Translation. La Haya: Kluwer Law International.

Tallon, Denis (1992): Damages, exemption clauses and penalties. American Journal of Comparative Law. 40:675-682.

Terral, Florence (2004) : L'empreinte culturelle des termes juridiques. Meta. 49(4):876-890.

VÁzquez Sotelo, José Luis (1994) : El ejercicio de la acción civil en el proceso penal. In: Enrique Ruiz Vadillo, dir. La responsabilidad civil derivada del delito:daño, lucro, perjuicio y valoración del daño corporal. Cuadernos de Derecho Judicial. Madrid: Consejo General del Poder Judicial. 18:103-137.

Vicente, Elena (2002): El daño. In: Luis Fernando Reglero, dir. Lecciones de responsabilidad civil. Cízur-Menor: Aranzadi, 71-89.

\section{ANNEXES}

Annexe 1 : Contextualisation des termes utilisés dans le droit espagnol pour évaluer les dommages

TABleau 5

Daño emergente et lucro cesante

\begin{tabular}{|l|l|l|}
\hline Source & Daño emergente & Lucro cesante \\
\hline $\begin{array}{l}\text { Código civil } \\
\text { español }\end{array}$ & Valor de la pérdida sufrida & $\begin{array}{l}\text { Ganancia que haya dejado de } \\
\text { obtener el acreedor }\end{array}$ \\
\hline Doctrine & $\begin{array}{l}\text { Pérdidas efectivamente sufridas y } \\
\text { desembolsos realizados en atención al } \\
\text { daño. }\end{array}$ & $\begin{array}{l}\text { Pérdidas efectivamente sufridas y } \\
\text { desembolsos realizados en atención } \\
\text { al daño. }\end{array}$ \\
\hline $\begin{array}{l}\text { Diccionario } \\
\text { de Derecho } \\
\text { (Ribó 1995) }\end{array}$ & $\begin{array}{l}\text { Menoscabo directo que sufre la } \\
\text { persona ofendida por un hecho ilícito } \\
\text { civil, en un valor que ya existe en su } \\
\text { patrimonio; se trata, pues, de un } \\
\text { daño o pérdida real y efectiva. }\end{array}$ & $\begin{array}{l}\text { Menoscabo directo que sufre la } \\
\text { persona ofendida por un hecho } \\
\text { ilícito civil, en un valor que ya } \\
\text { existe en su patrimonio; se trata, } \\
\text { pues, de un daño o pérdida real y } \\
\text { efectiva. }\end{array}$ \\
\hline
\end{tabular}




\begin{tabular}{|l|l|l|}
\hline $\begin{array}{l}\text { Black's Law } \\
\text { Dictionary } \\
\text { (Garner 2004) }\end{array}$ & $\begin{array}{l}\text { Damnum Emergens } \\
\text { An actual realized loss (Such as a } \\
\text { decline in the value of property). Is a } \\
\text { positive damage; consequential loss. }\end{array}$ & $\begin{array}{l}\text { Lucrum cessans } \\
\text { Expected future loss (Such as loss of } \\
\text { profit) } \\
\text { (p. 967) }\end{array}$ \\
$\begin{array}{ll}\text { It may be immediate (my slave is } \\
\text { killed or has lost an eye) or } \\
\text { consequential (I have lost his } \\
\text { services, I have incurred medical } \\
\text { expenses or the rest of the troupe is } \\
\text { less valuable in consequence of his } \\
\text { death or injury) } \\
\text { (p. 420) }\end{array}$ & \\
\hline
\end{tabular}

Annexe 2: Significations des termes spécifiques de damages et contextualisation

Tableau 6

Special damages et general damages

\begin{tabular}{|l|l|l|}
\hline Source & Special Damages & General damages \\
\hline Case law & $\begin{array}{l}\text { Cases where there were special and } \\
\text { extraordinary circumstances beyond the } \\
\text { reasonable prevision of the parties. To } \\
\text { be recovered they must be } \\
\text { communicated by and between the } \\
\text { parties Maranto v. Goodyear Tire \& } \\
\text { Rubber Co., 25,114 (La.App.2d } \\
\text { Cir.5/10/95), 661 So.2d 503 }\end{array}$ & $\begin{array}{l}\text { General damages are those } \\
\text { which cannot be fixed with } \\
\text { pecuniary exactitude. }\end{array}$ \\
\hline $\begin{array}{l}\text { Black's Law } \\
\text { Dictionary } \\
\text { (Garner 2004) }\end{array}$ & $\begin{array}{l}\text { Damages that are alleged to have been } \\
\text { sustained in the circumstances of a } \\
\text { particular wrong. Must be specifically } \\
\text { claimed and proved. }\end{array}$ & $\begin{array}{l}\text { Damages that the law presumes } \\
\text { follow from the type of wrong } \\
\text { complained of. General damages } \\
\text { do not need to be specifically } \\
\text { claimed or proved to have been } \\
\text { sustained. Also termed direct } \\
\text { damages; necessary damages }\end{array}$ \\
\hline $\begin{array}{l}\text { Diccionario de } \\
\text { Términos } \\
\text { Jurídicos } \\
\text { (Alcaraz et } \\
\text { Hugues 2001) }\end{array}$ & $\begin{array}{l}\text { Daños cuantificables, daños susceptibles } \\
\text { de comprobación y cálculos, } \\
\text { compensación por daños concretos que } \\
\text { se pueden precisar de forma clara, los } \\
\text { daños que no se pueden precisar de } \\
\text { forma clara se indemnizan de acuerdo } \\
\text { con lo que determina al ley se llaman } \\
\text { actual o general compensatory damages }\end{array}$ & $\begin{array}{l}\text { Daños efectivos, indemnización } \\
\text { compensatoria por daños } \\
\text { directos, generales o } \\
\text { efectivamente causados, de } \\
\text { acuerdo con lo que la ley estima } \\
\text { que el agraviado debe recibir; } \\
\text { este tipo de indemnización } \\
\text { también se llama actual/ } \\
\text { compensatory damages. }\end{array}$ \\
\hline $\begin{array}{l}\text { Diccionario } \\
\text { Jurídico } \\
\text { (Cabanellas et } \\
\text { Hoague 1993) }\end{array}$ & $\begin{array}{l}\text { Daños que son la consecuencia posible } \\
\text { pero no necesaria de una conducta, que } \\
\text { deben, por lo tanto, ser objeto de prueba } \\
\text { específica }\end{array}$ & \\
\hline
\end{tabular}


Tableau 7

Consequential damages et consequential loss

\begin{tabular}{|c|c|c|}
\hline Sources & Consequential damages & Consequential loss \\
\hline Statute law & $\begin{array}{l}\text { Art. 2- 715,1 UCC: any loss resulting } \\
\text { from general or particular requirements } \\
\text { and needs of which the seller at the time } \\
\text { of contracting had reason to know and } \\
\text { which could not reasonably be } \\
\text { prevented by cover or otherwise. } \\
\text { UCITA: Section 102: any loss resulting } \\
\text { from general or particular requirements } \\
\text { and needs of which the breaching party } \\
\text { at the time of contracting had reason to } \\
\text { know and which could not reasonably } \\
\text { be prevented; and any injury to an } \\
\text { individual or damage to property other } \\
\text { than the subject matter of the } \\
\text { transaction proximately resulting from } \\
\text { breach of warranty. The term does not } \\
\text { include direct damages or incidental } \\
\text { damages. }\end{array}$ & \\
\hline $\begin{array}{l}\text { Black's Law } \\
\text { Dictionary } \\
\text { (Garner 2004) }\end{array}$ & $\begin{array}{l}\text { Losses that do not flow directly and } \\
\text { immediately from an injurious act, but } \\
\text { that result indirectly from the act }\end{array}$ & $\begin{array}{l}\text { A loss arising from the results } \\
\text { of damage rather than from } \\
\text { the damage itself. A } \\
\text { consequential loss is } \\
\text { proximate when the natural } \\
\text { and probable effect of the } \\
\text { wrongful conduct, under the } \\
\text { circumstances, is to set in } \\
\text { operation the intervening } \\
\text { cause from which the loss } \\
\text { directly results. When the } \\
\text { loss is not the natural and } \\
\text { probable effect of the } \\
\text { wrongful conduct, the loss is } \\
\text { remote. Also termed indirect } \\
\text { loss. Cf. direct loss. }\end{array}$ \\
\hline $\begin{array}{l}\text { Diccionario de } \\
\text { Términos } \\
\text { Jurídicos } \\
\text { (Alcaraz et } \\
\text { Hugues 2001) }\end{array}$ & $\begin{array}{l}\text { Consecuente, perjuicios; daños } \\
\text { emergentes, consecuentes o especiales. } \\
\text { V. special damages }\end{array}$ & \\
\hline $\begin{array}{l}\text { Diccionario } \\
\text { Jurídico } \\
\text { (Cabanellas et } \\
\text { Hoague 1993) }\end{array}$ & $\begin{array}{l}\text { Daño indirecto; indemnización del } \\
\text { daño indirecto }\end{array}$ & \\
\hline
\end{tabular}


Tableau 8

Incidental damages

\begin{tabular}{|l|l|}
\hline Sources & Incidental damages \\
\hline Statute law & $\begin{array}{l}\text { 2-715, 1 UCC expenses reasonably incurred in inspection, receipt, } \\
\text { transportation and care and custody of goods rightfully rejected, any } \\
\text { commercially reasonable charges, expenses or commissions in } \\
\text { connection with effecting cover and any other reasonable expense } \\
\text { incident to the delay or other breach. }\end{array}$ \\
\hline \begin{tabular}{l} 
Black's Law $\begin{array}{l}\text { Dictionary (Garner } \\
\text { 2004) }\end{array}$ \\
\hline $\begin{array}{l}\text { Diccionario de } \\
\text { Términos Jurídicos } \\
\text { (Alcaraz et Hugues } \\
\text { 2001) }\end{array}$
\end{tabular} & $\begin{array}{l}\text { Losses reasonably associated with or related to actual damages. A } \\
\text { seller's commercially reasonable expenses incurred in stopping } \\
\text { delivery or in transporting and caring for goods after a buyer's breach. }\end{array}$ \\
\hline $\begin{array}{l}\text { Diccionario Jurídico } \\
\text { (Cabanellas et } \\
\text { Hoague 1993) }\end{array}$ & Pérdidas suplementarias ocasionadas al demandante \\
\hline
\end{tabular}

Tableau 9

Direct damages et indirect damages

\begin{tabular}{|l|l|l|}
\hline Source & Direct damages & Indirect damages \\
\hline Case law & $\begin{array}{l}\text { The cost to cure, } \\
\text { remedy or remediate } \\
\text { the physical damage } \\
\text { or situation }\end{array}$ & $\begin{array}{l}\text { Indirect damages, which has sometimes } \\
\text { been referred to as stigma damages. Indirect } \\
\text { damages (a form of economic loss) is defined } \\
\text { as all loss of income and value of a property, } \\
\text { from the moment of the discovery of the } \\
\text { situation until the property has returned to } \\
\text { its nominal market value, excluding only the } \\
\text { direct cost of remediation or repair }\end{array}$ \\
\hline $\begin{array}{l}\text { Black's Law } \\
\text { Dictionary (Garner }\end{array}$ & See general damages & $\begin{array}{l}\text { Le terme n'apparait pas. Nous trouvons la } \\
\text { référence à indirect losses: see } \\
\text { consequential loss }\end{array}$ \\
\hline $\begin{array}{l}\text { Diccionario de } \\
\text { Términos Jurídicos } \\
\text { (Alcaraz et Hugues } \\
\text { 2001) }\end{array}$ & $\begin{array}{l}\text { Daños generales o } \\
\text { directos }\end{array}$ & \\
\hline
\end{tabular}


TABLeau 10

Punitive damages

\begin{tabular}{|c|c|}
\hline Source & Punitive damages \\
\hline $\begin{array}{l}\text { Black's Law } \\
\text { Dictionary } \\
\text { (Garner 2004) }\end{array}$ & $\begin{array}{l}\text { Damages awarded in addition to actual damages when the defendant } \\
\text { acted with recklessness, malice, or deceit. Punitive damages, which are } \\
\text { intended to punish and thereby deter blameworthy conduct, are generally } \\
\text { not recoverable for breach of contract. }\end{array}$ \\
\hline $\begin{array}{l}\text { Diccionario de } \\
\text { Términos } \\
\text { Jurídicos } \\
\text { (Alcaraz et } \\
\text { Hugues 2001) }\end{array}$ & $\begin{array}{l}\text { Daños punitivos, daños ejemplares. A court may award punitive damages } \\
\text { if it comes to the conclusion that the defendant has calculated that the } \\
\text { advantage to him of committing the tort would outweigh the damages } \\
\text { against him. Cf. exemplary damages }\end{array}$ \\
\hline $\begin{array}{l}\text { Diccionario } \\
\text { Jurídico } \\
\text { (Cabanellas et } \\
\text { Hoague 1993) }\end{array}$ & Daños punitorios \\
\hline
\end{tabular}

TABLEAU 11

Exemple de contextualisation du terme damages

\section{Indemnización de daños y perjuicios}

CISG : Article 74

Damages for breach of contract by one party consist of a sum equal to the loss, including loss of profit, suffered by the other party as a consequence of the breach.

\section{Daño}

UCITA: Section 102

Any injury to an individual or damage to property other than the subject matter of the transaction proximately resulting from breach of warranty. 